\title{
Flexibilidad cognitiva, personalidad proactiva y aprendizaje en adolescentes: desde una perspectiva cualitativa
}

Botero-Arango, Yesica Daniela ${ }^{1}$; Castro-Muñoz, Melissa ${ }^{1\left({ }^{*}\right)}$; Gallego-Tavera, Sindy Yuliana ${ }^{1}$

${ }^{1}$ Vicerrectoría de Investigaciones, Grupo GORAS, Universidad Católica Luis Amigó, Facultad de psicología Medellín, Colombia

\section{RESUMEN}

En el complejo mundo de las relaciones interpersonales, el individuo se enfrenta a un proceso como la adolescencia que puede ser descrita como un periodo que transcurre entre los cambios biológicos, físicos y psicológicos. Dado que es donde cambian los roles de niño a adolescente y donde se establece la identidad; estos procesos vienen de la mano del aprendizaje, apelando a un proceso de aprendizaje y flexibilidad cognitiva, en donde se aprecian diferentes teorías sobre este mismo fenómeno, en el cual el aprendizaje por imitación es determinante para la identificación con pares, teniendo en cuenta todos los procesos nombrados anteriormente, estos encuentran relación con "la personalidad proactiva que está vinculada a los intentos activos que realiza el individuo para efectuar cambios en su ambiente, solucionar problemas interpersonales, generar respuestas funcionales y considerar las consecuencias positivas" (Zampetakis, 2008, p. 155).

Palabras clave: Flexibilidad cognitiva. Adolescencia. Aprendizaje. Personalidad proactiva.

\section{Cognitive flexibility, proactive personality and learning in adolescents: from a qualitative perspective}

\section{ABSTRACT}

In the complex world of interpersonal relationships, the individual faces a process such as adolescence that can be described as a period between biological, physical and psychological changes. Since it happens where the roles change from child to adolescent, and where identity is established, these processes come along with learning, leading to a decision - making process and cognitive flexibility, where different theories can be appreciated. In this transformation, learning by imitation is decisive for identification with peers, taking into account all the processes mentioned above are related to "the proactive personality that is linked to the active attempts made by the individual to effect changes in their environment, solve interpersonal problems, generate functional responses and consider the positive consequences" (Zampetakis, 2008, p. 155).

Keywords: Cognitive flexibility. Adolescence. Learning. Proactive personality.

Recibido: 28/04/2021 Aceptado:02/06/2021

Correspondencia: $\left({ }^{*}\right)$ melissa.castromu@amigo.edu.co 


\section{INTRODUCCIÓN}

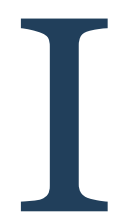

ndagar acerca de la flexibilidad cognitiva, la personalidad proactiva y el aprendizaje en la adolescencia, es pertinente en la actualidad ya que, es una etapa donde no solo ocurren cambios a nivel físico, sino también emocional, psicológicos, cognitivos y lo que es aún más relevante a nivel cerebral, no obstante empiezan a evidenciarse cambios emocionales y de adaptación, es aquí donde se da un antagonismo en el relacionamiento con los padres, empiezan a evidenciarse cambios en el humor, hay una preocupación importante por el aspecto físico, incrementa el interés por la sexualidad y por generar vínculos con pares, surge la necesidad de conformar grupos sociales con los cuales se forja la identidad y nace la necesidad de tener espacios íntimos.

Por otro lado, los adolescentes buscan adaptarse al contexto, ser aprobados y no segregados, esta adaptación engloba tres factores relevantes que se analizaron en el curso, las cuales fueron, identificar si existe relación entre la flexibilidad cognitiva, personalidad proactiva y aprendizaje en los adolescentes.

Podría definirse la flexibilidad cognitiva como la capacidad que tiene una persona de adaptarse a los diferentes contextos en los que está inmerso y tomar decisiones acertadas. La personalidad proactiva, está relacionada con el actuar de manera rápida y estratégica con la intención de predecir problemas y actuar incluso antes de que estas sucedan. La toma de decisiones en los adolescentes es un proceso importante en donde, se decide que se quiere hacer, alcanzar y elegir los medios para la solución de los problemas.

Actualmente son escasos los estudios que relacionan flexibilidad cognitiva, personalidad proactiva y aprendizaje en adolescentes, es por esto que surge la necesidad de indagar la relación entre estas tres categorías, a su vez que explorar los procesos psicológicos que implica dicha relación, por lo anterior se plantean los siguientes objetivos; conocer cuáles son las características de la personalidad proactiva, flexibilidad cognitiva y aprendizaje en los adolescentes, así mismo explorar la influencia de la personalidad proactiva y la flexibilidad cognitiva respecto a los adolescentes y por último describir los elementos teóricos que fundamentan la relación entre flexibilidad cognitiva, personalidad proactiva y aprendizaje.

Finalmente, la pretensión de este manuscrito es proporcionar elementos y datos relevantes a la comunidad académica e identificar los beneficios y contratiempos de dicha investigación permitiendo una contribución de la conceptualización de la flexibilidad cognitiva, la personalidad proactiva y aprendizaje en adolescentes.

\section{MARCO TEÓRICO}

Según Diz (2013), la adolescencia se traza como el espacio que transcurre entre la inauguración de la pubertad y el final del crecimiento, mientras que la pubertad es un periodo de cambios biológicos; la adolescencia es una construcción psicosocial ( $p$. 88). Esta es una etapa donde transcurren grandes cambios a nivel, físico emocional y psíquicos, permitiendo que sea un periodo donde el niño pueda prepararse para ser un adulto, así mismo es una etapa donde madura la capacidad de raciocinio y capacidad emocional, las cuales son fases importantes que entran en la maduración cerebral del joven.

De la misma forma, Viner, et al. (2017) hacen hincapié en mencionar que la pubertad es el efecto de un progreso orgánico, adelanto cerebral, conocimiento, reconocimiento sexual y capacidad reproductiva. Los cambios que sufre el niño (pubertad) llegan de forma abrupta lo que origina un momento estresante y pavoroso para los adolescentes y su núcleo familiar, puesto que encarna una etapa de trascendentales cambios emocionales y físicos como lo menciona Tang et al. (2020). Por consiguiente, el niño y su foco familiar no se encuentran dispuestos para los cambios que advierte a lo largo de esta etapa; el joven se encuentra en una metamorfosis en la cual deja hábitos, conoce y se vincula con otras personas, se atañe por otras cosas, todo esto viene influido por componentes hereditarios, circunstanciales y 
nutricionales que son explicativos en el progreso de la pubertad. Kota \& Ejaz (2020).

Orenstein \& Lewis (2020) Hacen énfasis en que la identidad es primordial en la adolescencia y que hace fragmento de la fase número cinco en la escala del desarrollo de Erik Erickson y es el espacio donde se presenta aprietos para fundar la identidad, el joven tiene en cuenta sus prácticas mientras está transversalizado por las expectativas sociales. Aquí el adolescente siente una fuerte necesidad de enfrentarse a las figuras vinculares o de autoridad (padres o cuidadores).

En otras palabras, refiere Prado \& Rodriguez (2001), "que en el adolescente hay un conflicto de identidad, por no hallar una respuesta a los canjes que se dan en su cuerpo, pero con el paso del tiempo les haya un recurso" Esto lo logra con ayuda de los grupos sociales en los que se ha integrado, gracias a la independencia que a alcanzado, lo que llevo a que encontrará sus límites, gustos, descubriera un sentido de lo que ocurrió en su cuerpo y el rol que está cumpliendo dentro de la sociedad y dentro de su núcleo familiar y como se ha de desenvolver en estos.

Por otro lado, Schunk (1997) "Un juicio trascendental para precisar el aprendizaje es el cambio en la capacidad de comportarse conductualmente. Se emplea el término aprendizaje cuando cualquiera se torna competente en crear algo diferente a lo que creaba antes" por consiguiente, es importante hablar de aprendizaje y tener en cuenta que el " constructivismo, cognitivismo y conductismo son las tres grandes teorías de aprendizaje" Siemens \& Fonseca (2007).

El objetivo del conductismo es conseguir, en este caso que el adolescente obtenga prácticas o habilidades, en las cuales se puede demostrar el cambio de conducta mientras que el cognitivismos expone que el aprendizaje se posee a través del tiempo y mediante la experiencia, mientras que el constructivismo bosqueja que el conocimiento se funda, busca interacción con el mundo exterior, es relevante expresar que hay otras perspectivas, también importantes que han aportado en el campo del aprendizaje como por ejemplo: "La taxonomía de
Bloom que instituyó una expresión común de modo que los materiales de aprendizaje lograrán compararse entre instituciones, esta taxonomía tiene los tres imperios de aprendizaje que son: cognitivo, afectivo y psicomotor" Orgill \& Nolin (2020). Para Bloom es significativo indicar que estos tres dominios permiten entender el aprendizaje en cualquier institución. En primer lugar, Soledad (2014) describe el objetivo de la psicología cognitiva, es estar al tanto, cómo el ser humano de cualquier edad adquiere información, como personifica la pesquisa en su cerebro y cómo concierta esta nueva información con otras averiguaciones existentes en su mente y cómo la rescata cuando lo necesita. (Cap.1) es decir que está implicado en las áreas del conocimiento desde la memoria, la percepción, atención, pensamiento, lenguaje y aprendizaje, a partir de esto explica la conducta que surge de asociaciones de estímulos respuestas.

En segundo lugar, se encuentra el dominio afectuoso, en este es ineludible definir el juicio emocional que es concebido como la cabida que tiene la persona para examinar sentimientos en sí mismo y en otros, Goleman (2001) permitiendo que sean más asertivos en la comunicación y socialización con otras personas, ya que el manejo de las emociones configura una herramienta indispensable para que los adolescentes puedan afrontar diferentes e importantes situaciones de la vida. Por otro lado, Fernández et al. (2010) asevera en este caso, desafiar el progreso biológico por el que pasa el niño, y plantearse los cambios psicológicos que advierte el adolescente, a partir de esto se funda que un adolescente que desenvuelve el aprendizaje socioemocional tiene la cabida de tener mejor relaciones consigo mismo y con el mundo, es asertivo y tiene un excelente rendimiento académico, sin embargo Sánchez \& Llera (2014), advierten que a menudo surgen dificultades a nivel escolar o conflictos de aprendizaje en la adolescencia. Es un período en que los factores socioemocionales y orgánicos alcanzan una gran notabilidad (p.36), pues como se ha mencionado anteriormente el adolescente está en una etapa de desarrollo cognitivo, emocional y psíquico, y está aprendiendo a convivir con estos.

Por su parte, Morales et al. (2018) nombran que los 
adolescentes hacen parte de su aprendizaje la imitación, no solo para llevar a cabo establecidas conductas sino también para buscar identificarse con algunos personajes que les auxilien a adaptarse al medio. Según, Bandura et al. (1977) el individuo observa y guarda en su memoria las imágenes que le atraen para luego reproducirlas en determinado contexto. Tello Terreros, (2013) menciona que en la etapa inicial la adolescencia suele buscar sus modelos de identificación en sus figuras significativas o en personajes, siguiendo programas de televisión para posteriormente continuar copiando sus conductas.

\section{Personalidad Proactiva.}

Astorne, M., \& Stefania, M. (2017) La personalidad proactiva, (como se citó en Crant (1995), hace énfasis al grado en que las personas están dispuestas a actuar de modo que influyan positivamente en su entorno; teniendo presente cualquier eventualidad que pueda generarse de manera inesperada $y$ promoviendo soluciones rápidas y eficaces, respondiendo a la demanda del problema. Por otro lado, "La proactividad está vinculada a los intentos activos que realiza el individuo para efectuar cambios en su ambiente" (Zampetakis, 2008, p. 155). Otros autores como Bateman y Crant (1993), afirman que las personas con personalidades proactivas tienden a tomar la iniciativa para influir y cambiar significativamente el entorno. En otras palabras, tener una personalidad proactiva puede ayudar a un individuo a liberar presiones situacionales, identificar oportunidades de ventaja, realizar movimientos proactivos y, por lo tanto, influir en el entorno para crear cambios significativos.

Junto con investigaciones previas sobre adaptabilidad ambiental (Crant, 1995, 2000) y proactividad, y estudios empíricos adicionales han confirmado que las personas proactivas tienen más probabilidades de lograr el éxito en el estudio, trabajo, vida personal, y por ende están más en sintonía con la necesidad de dominio, logros, autonomía, confianza en sí mismos y conciencia. (Claes et al., 2005). De esta manera, los adolescentes con personalidad proactiva aprenden a hacer una pausa, a ejercer el control y pensar de qué modo reaccionar, y tomar decisiones más prudentes y asertivas. La personalidad proactiva permite utilizar todas las herramientas humanas que cada uno tiene a su alcance, algunas de estas herramientas son la imaginación la conciencia, la voluntad independiente y el autoconocimiento, estas herramientas permiten una mejor ilustración para decidir positivamente que es lo que se debe hacer.

Del mismo modo, las personas proactivas toman decisiones con base en los valores, lo que los lleva en la mayoría de las veces, a realizar las cosas y a tomar decisiones pertinentes y a conciencia, es decir pensando en las consecuencias y resultados de las diferentes acciones que se puedan llevar a cabo. Piensan antes de actuar, reconocen que no pueden controlar todo lo que les sucede, pero sí pueden controlar lo que pueden hacer al respecto. (Covey, 2015, p. 49).

\section{Flexibilidad Cognitiva y Funciones Ejecutivas.}

La flexibilidad cognitiva es definida como una conducta abierta, ordenada, sistematizada, y por ende, dará una respuesta rápida a estímulos de clasificación (Seisdedos, 2000). Según Floresco (2011), existen muchas regiones del cerebro que trabajan en conjunto para crear una conducta adaptable frente ambientes demandantes y cambiantes; en la literatura relacionada a la variable se define que los sujetos con flexibilidad cognitiva son capaces de solucionar problemas como respuesta adaptativa a los cambios que se producen en una determinada situación. En relación a lo anterior, la flexibilidad cognitiva permite adaptar nuestra conducta y pensamiento a situaciones novedosas, es un camino para el reajuste no solo de la conducta sino también del pensamiento y las opiniones. La flexibilidad cognitiva le ofrece a la persona tener una visión desde diferentes perspectivas, acompañada de poder encontrar varias soluciones al mismo tiempo en el que se presente un problema o situación que lo amerite, permite por otro lado, tolerar mejor los errores o coyunturas que se puedan llegar a presentar, también permite consentir mejor los cambios repentinos de planes.

Del mismo modo, la flexibilidad cognitiva puede considerarse como la capacidad para adaptar los desempeños a las condiciones ambientales frente a 
una tarea (Cañas, Quesada, Antoli, Fajardo, \& Salmerón, 2005, en Ison, n.d.) o bien como un componente de las funciones ejecutivas (Sánchez-Carpintero \& Narbona, 2004). Existe consenso en considerar como componentes de las funciones ejecutivas a: la capacidad de planificación de conductas dirigidas a una meta, la programación de acciones necesarias para alcanzar dicha meta, la monitorización de la ejecución, la capacidad de controlar la interferencia de estímulos irrelevantes, la flexibilidad para corregir errores, incorporar conductas nuevas y para finalizar una tarea cuando se ha concluido.

Seguidamente, encontramos que según los autores la flexibilidad cognitiva es aquella capacidad mental para cambiar de pensamiento alrededor de dos conceptos diferentes y pensar en múltiples cosas, en donde enfatiza la posibilidad del traslado de conocimientos, capacidades y habilidades más allá de su situación de aprendizaje inicial debido a que se extrapola la información anterior y se observa desde varias perspectivas novedosas, logrando utilizar el conocimiento o información en momentos posteriores (Valverde, 2014).

La flexibilidad cognitiva "se ha descrito con más detalle cómo; la capacidad de ajustar el pensamiento de situaciones anteriores a actuales, así como la habilidad de superar respuestas o pensamientos que se han vuelto frecuentes y se adaptan a nuevas situaciones" (Moore \& Malinowski, 2009 p. 177).

\section{Adaptación y adolescencia.}

Dentro del ciclo vital humano, la adolescencia es una etapa que se encuentra caracterizada por una serie de cambios biológicos, psicológicos y sociales que preceden a la etapa adulta y que tienen que ver con la búsqueda de autonomía, adaptación en su entorno, identidad propia, diferenciación del medio familiar, sentido de la vida y búsqueda de pertenencia con los pares. Biológicamente, se dice que la adolescencia comienza con la pubertad, que ocurre aproximadamente entre los 10 y 12 años, cuando se adquiere la capacidad para reproducirse, y termina alrededor de los 17 o 19 años; no obstante, su base física comienza antes y sus efectos psicológicos pueden perdurar mucho tiempo después. (Aragón et al., 2012). Es por lo anterior que la adolescencia y la adaptación dentro de este espacio del ciclo vital van de la mano, en esta etapa de cambio físico, psicológico y social el adolescente se encuentra fuertemente presionado por el ambiente, adquiriendo habilidades para la interacción deseable con otros y lo que busca la adaptación es incorporarlo al entorno al que hace parte de manera adecuada y armónica.

Davidoff (1979, citado en García \& Magaz, 1998) señala que una persona bien adaptada tiene sentimientos positivos de sí misma y se considera competente y con éxito en la vida, muestra un sentido de autonomía e independencia, es activa, laboriosa, muestra grandes niveles de creatividad, proactividad y resolución de problemas en caso de presentarse en el entorno o lugar en el que se encuentra y suficiente enérgica en la consecución de sus intereses, o metas, se relaciona bien, armónicamente con los demás y se siente satisfecha de su vida, disfruta de ella y no la abruman los problemas. Por lo anterior, se considera entonces que la adaptación humana consiste en un doble proceso: primero en un ajuste de la conducta del individuo a sus propios deseos, gustos, preferencias y necesidades y segundo un ajuste de tal conducta a las circunstancias del entorno en que vive, es decir a las normas, deseos, valores, gustos, y necesidades de las personas con las que interactúa ocasional o habitualmente. (García \& Magaz, 1998, p. 13).

Davidoff (1979), en su libro "Introduction to psychology" realiza una amplia exploración del concepto resolviendo que el individuo bien adaptado:

1. Tiene sentimientos positivos acerca de sí mismo, y se considera competente y con éxito en la vida.

2. Muestra un sentido de autonomía e independencia.

3. Es activo, laborioso y enérgico en la consecución de sus intereses.

4. Se relaciona bien, armónicamente, con los demás.

5. Se siente satisfecho de su vida, disfruta de ella y no le abruman los problemas. 
Es decir, que el adolescente hace un intento por acomodarse y ajustarse a las condiciones y circunstancias que se le presentan, la adaptación también es una parte del individuo que se ajusta a las realidades, existe entonces un deseo de encajar en el adolescente, hay un anhelo latente de equilibrio interno entre sus deseos y necesidades.

Los conceptos anteriores sobre adaptación hacen referencia a la necesidad que tiene el adolescente de lograr un ajuste entre sus propias características personales y sociales, incluyendo sus necesidades, y las demandas del medio donde interactúa. Como ya se mencionó, una de las etapas del ciclo vital en la que las personas intentan lograr un acuerdo armónico entre ellos mismos y su medio ambiente, es especialmente la adolescencia. (Bosques et al., 2008).

\section{METODOLOGÍA}

El reciente trabajo se desarrolló bajo una sistemática de revisión bibliográfica que permitan el análisis y documentación del material más importante en bases de datos, como: PubMed, Google academic, Ebsco, Redalyc, SciELO, Pepsic y Dialnet; concibiendo el uso de los siguientes juicios de búsqueda " adolescencia" "aprendizaje" " personalidad proactiva" "flexibilidad cognitiva" "funciones ejecutivas " "adaptación y adolescencia" Con la intención de examinar la obtención bibliográfica que ha sido desarrollada en los últimos años, en pro de indagar y dar respuesta a las hipótesis establecidas por la presente exploración. Por este motivo, el actual trabajo al tener en cuenta que no hay muchas investigaciones sobre esta temática en el idioma español, con el propósito de analizar la producción bibliográfica y encontrar relación entre las variables anteriormente nombradas, permitiendo así el ahondamiento en dicha temática que posibilita el conocimiento y reconocimiento de las variables a trabajar desde una perspectiva neuropsicológica.

\section{DISCUSIÓN}

La adolescencia está impregnada de diversos factores que aportan al desarrollo de dicha etapa, uno de estos factores es el aprendizaje, Schunk (1997) menciona que el aprendizaje es el cambio en la capacidad de comportarse conductualmente, hacer cosas que no se hacían antes, como por ejemplo adquirir hábitos. Por otra parte, dice Siemens \& Fonseca (2007) que el constructivismo, cognitivismo y conductismo son las tres grandes teorías de aprendizaje" y que en ella se encuentra el sentido de lo que compone en sí el aprendizaje en las diversas etapas evolutivas.

Para complementar se le suma la "La proactividad que está vinculada a los intentos activos que realiza el individuo para efectuar cambios en su ambiente" (Zampetakis, 2008, p. 155) cuando un adolescente genera cambios en su ambiente, notablemente está ejerciendo cambios en su conducta producto del aprendizaje y la experiencia, por otro lado, dentro de los procesos de aprendizaje podría considerarse necesario tener presente la flexibilidad cognitiva definida como: "la capacidad de ajustar el pensamiento de situaciones anteriores a actuales, así como la habilidad de superar respuestas o pensamientos que se han vuelto frecuentes y se adaptan a nuevas situaciones" (Moore \& Malinowski, 2009 p. 177). Es decir, que lo anterior como un todo integrado en la adolescencia, permite generar cambios en el comportamiento en el ámbito conductual, la toma de decisiones y permite tener posibilidades de cambio en el ambiente.

En este orden, este articulo recopila no solo el aprendizaje propuesto por los autores mencionados, sino también la flexibilidad cognitiva, la adaptación en la adolescencia y la proactividad en relación a dicha categoría (aprendizaje).

\section{CONCLUSIONES}

La revisión bibliográfica desarrollada en este escrito posibilito comprender los significados y relación teórica que existen entre la flexibilidad cognitiva, 
personalidad proactiva y aprendizaje en adolescentes, en los cuales se genera procesos interactivos y de intercambio entre las diferentes variables.

Es importante señalar que estudios empíricos adicionales han confirmado que las personas proactivas, con alta flexibilidad cognitiva y con apertura al aprendizaje tienen más probabilidades de lograr el éxito en el estudio, trabajo, vida personal, y por ende están más en sintonía con la necesidad de dominio, logros, autonomía, confianza en sí mismos y conciencia. (Claes et al., 2005).

Finalmente, es por ello importante resaltar la necesidad de desarrollar apuestas teóricas, lecturas contextuales y metodológicas que permitan ampliar y favorecer la construcción de significados de las variables aquí utilizadas y sus múltiples relaciones en la adolescencia; la serie de cambios biológicos, psicológicos y sociales que se producen durante este ciclo y transición a la etapa adulta, en la cual es de suma importancia la conducta y el contexto ya que es aquí donde la proactividad y la flexibilidad cognitiva toman importancia y coincide con el aprendizaje. 


\section{REFERENCIAS}

Aragón Borja, Laura Edna, \& Bosques, Elena (2012). ADAPTACIÓN FAMILIAR, ESCOLAR Y PERSONAL DE ADOLESCENTES DE LA CIUDAD DE MÉXICO. Enseñanza e Investigación en Psicología, 17(2),263-282. [fecha de Consulta 16 de Octubre de 2020]. ISSN: 0185-1594. Disponible en: https://www.redalyc.org/articulo.oa?i$d=292 / 29224159002$

Bateman, T. S., and Crant, J. M. (1993). The proactive component of organizational behavior: a measure and correlates. J. Organ. Behav. 14, 103-118. https://doi: 10.1002/job.4030140202.

Bandura, Albert, y Walters, (1977), R.H. Aprendizaje social y desarrollo de la personalidad, Madrid: Alianza Ed

Bosque Fuentes, Ana Elena del, \& Aragón Borja, Laura Edna. (2008). Nivel de adaptación en adolescentes mexicanos. Interamerican Journal of Psychology, 42(2), 287-297. Recuperado em 16 de octubre de 2020, de http://pepsic.bvsalud.org/scielo.php?script=sci_arttext\&pi$d=$ S0034-96902008000200010\&lng=pt\&tlng=es

Carbajal Sifuentes, P. L. (2017). Personalidad proactiva y capacidad emprendedora de los trabajadores de una empresa dedicada al rubro de telecomunicaciones en el distrito de Independencia, 2017.Recuperado por: http://repositorio.ucv. e d u . p e / b i t s t r e a m / h a n dle/20.500.12692/3195/Carbajal_SPL.pdf?seque nce $=1 \&$ isAllowed $=y$

Carena, S. C., Pisano, M. M., \& Tessio Conca, A. (2012). Ser joven en América latina a comienzos del tercer milenio. Diálogos Pedagógicos, 7(13), 11-33. Recuperado a partir de http://revistas.bibdigital.uccor.edu.ar/index.php/dialogos/article/view/118.

Crant, J., M. (1995). The Proactive Personality Scale and objective job performance among real estate agents. Journal of Applied Psychology, 80(4), 532-537. https://doi.org/10.1037/0021-9010.80.4.532
Crant, J., M. (2000). Proactive behavior in organizations. Journal of Management, 26(3), 435-462. https://doi.org/10.1177/014920630002600304

Claes, R., Beheydt, C., \& Lemmens, B. (2005). Unidimensionality of Abbreviated Proactive Personality Scales across Cultures. Applied Psychology: An International Review, 54(4), 476-489. $\mathrm{h} t \mathrm{t} \quad \mathrm{s}:$ / / d o i . o r g/10.1111/j.1464-0597.2005.00221.x

Covey, S. (2015). Los 7 hábitos de los adolescentes altamente efectivos: En la era digital. Grijalbo.

Davidoff, L. (1979). Introducción a la psicología. México: McGraw-Hill.

Diz, J. I. (2013). Desarrollo del adolescente: aspectos físicos, psicológicos y sociales. Pediatr Integral, 17(2), 88-93

Fernández, O., Luquez, P., \& Leal, E. (2010). Procesos socio-afectivos asociados al aprendizaje y práctica de valores en el ámbito escolar. Telos, 12(1), 63-78. Disponible en: https://www.redalyc.org/articulo.oa?id=99312518005.

Floresco, S. (2011). Neuronal circuits underlying flexibility. Multiple brain regions work together to adapt behavior to changing environment. Disponible en: http://www.apa.org/science/about/psa/2011/04/neural-circuits.aspx.

Goleman, Daniel (2001). La práctica de la inteligencia emocional (decimocuarta edición). Editorial Kairós. España.

Grant, J. (1995). The Proactive Personality Scale and objective job performance among real estate agents. Journal of Applied Psychology , 80, 532 7. Recuperado de: http://psycnet.apa.org/index.cfm ? $\mathrm{f} a=b u y$. op tion ToB uy \&ui $d=1995-42526-001$

Henderson, S. Aspectos psicológicos de la adolescencia normal. S. HENDERSON, Jornadas sobre" Adolescencia

Ison, M. S. (n.d.). Flexibilidad cognitiva y solución de problemas. Unpublished manuscript. 
Kidwell, J. S., Dunham, R. M., Bacho, R. A., Pastorino, E., \& Portes, P. R. (1995). Adolescent identity exploration: a test of Erikson's theory of transitional crisis. Adolescence, 30(120), 785-793.

Kota, A., S., \& Ejaz, S. (2020). Precocious Puberty. In Stat Pearls. Stat Pearls Publishing.

Kuniyoshi, Y. (2015). Learning from Examples: ImitationLearning and Emerging Cognition. In G. Cheng PhD (Ed.), Humanoid Robotics and Neuroscience: Science, Engineering and Society. CRC Press/Taylor \& Francis.

Lucero, J. C. V., Barajas, J. A., Muñiz, J. G., González, C. M., Delgado, R. M., \& Alvarado, I. U. (2014). Influencia del clima familiar en el proceso de adaptación social del adolescente [Influence of family environment in the adolescent's social adaptation process]. Psicología desde el Caribe, 31(2), 207-222. https://doi.org/10.14482/psdc.31.2.6127.

Morales, Soledad, Cabrera, Magela, \& Rodríguez, Gabriela. (2018). Estrategias de aprendizaje informal de habilidades transmedia en adolescentes de Uruguay. Comunicación y sociedad, (33), 65-88. https://doi.org/10.32870/cys.v0i33.7007

Moore, A., \& Malinowski, P. (2009). Meditation, mindfulness and cognitive flexibility. Consciousness and cognition, 18(1), 176-186. https://doi.org/10.1016/j.concog.2008.12.008

Orenstein, G. A., \& Lewis, L. (2020). Erikson's Stages of Psychosocial Development. In Stat Pearls. StatPearls Publishing.

Orgill, B. D., \&Nolin, J. (2020). Learning Taxonomies in Medical Simulation. In StatPearls. StatPearls Publishing.

Prado, C. A. V., \& Rodríguez, R. E. N. (2001). aportaciones de la teoría psicoanalítica al desarrollo de la adolescencia: la intervención en el aula.
Sánchez, L. P., \& Llera, J. B. (2014). Estrategias de aprendizaje. Función y diagnóstico en el aprendizaje adolescente. Padres y Maestros/Journal of Parents and Teachers, (358), 34-39. Recuperado a partir de https://revistas.comillas.edu/index.php/padresymaestros/article/view/4086.

Sánchez-Carpintero, R., \& Narbona, J. (2004). El sistema ejecutivo y las lesiones frontales en el niño. Revista de Neurología, 39(2), 188- 191. https://doi.org/10.33588/rn.3902.2004251.

Seisdedos, N. (2000). Cambios. Test de flexibilidad cognitiva. ( $3^{\circ}$ ed.). Madrid: Tea Editores.

Siemens, G., \& Fonseca, D.E. (2007). CONECTIVISMO: UNA TEORÍA DE APRENDIZAJE PARA LA ERA DIGITAL.

Soledad, B. J. (2014). Habilidades cognitivas básicas: formación y deterioro. Editorial UNED.

Solano, A. C. (2011). Estrategias de aculturación y adaptación psicológica y sociocultural de estudiantes extranjeros en la Argentina. Interdisciplinaria, 28(1), 115-130. Disponible en: https://www.redalyc.org/articulo.oa?i$d=18022327007$

Schunk, D. H. (1997). Teorías del aprendizaje. Pearson education.

Tang, C., Zafar Gondal, A., \& Damian, M. (2020). Delayed Puberty. In StatPearls. StatPearls Publishing.

Tello Terreros, D. F. (2013). Programa de prevención debido a la incidencia del aprendizaje por imitación en el consumo de alcohol, en la relación padres e hijos (Bachelor'sthesis, Universidad del Azuay).

Uribe Aramburo, N. I. (2011). Adolescencia y ritos de transición: una articulación del psicoanálisis post freudiano y lacaniano. Disponible en: https://www.redalyc.org/articulo.oa?i$\mathrm{d}=497856287005$ 


\section{REFERENCIAS}

Viner, R. M., Allen, N. B., \&Patton, G. C. (2017). Puberty, Developmental Processes, and Health Interventions. In D. Bundy (Eds.) et. al., Child and Adolescent Health and Development. (3rd ed.). The International Bank for Reconstruction and Development / The World Bank. https://doi.org/10.1596/978-1-4648-0423-6_ch9

Valverde, J. (2014). Moocs: Una visión crítica desde las ciencias de la educación profesorado, Revista de Currículum y Formación de Profesorado, 18 (1), 93-111. Recuperado por: http://www.redalyc.org/pdf/567/56730662007.pdf

Vericat, A., \& Orden, A. B. (2010). Herramientas de screening del desarrollo psicomotor en Latinoamérica. Revista chilena de pediatría, 81(5), 391-401. https://dx.doi.org/10.4067/S0370-41062010000500002

Zampetakis, L. A. (2008). The role of creativity and proactivity on perceived entrepreneurial desirability. Thinking Skills and Creativity, 3(2), 154-162. https://doi.org/10.1016/j.tsc.2008.07.002 